\title{
Calculation and Simulation of the Ground State Properties of Copper-Nickel Alloys
}

\author{
Huaqing Pan', Tieyu Lü², Jincheng Zheng ${ }^{2}$, and Shuo Lin $^{3}$ \\ ${ }^{1}$ Department of Mechanical Engineering, Shangrao Vocational and Technical College, Shangrao, China, \\ 334100 \\ ${ }^{2}$ Department of Physics, Xiamen University, Xiamen, China, 361005 \\ ${ }^{3}$ College of Physics and Information Engineering, Minnan Normal University, Zhangzhou, China, 363000
}

Keywords: Copper-Nickel Alloys; ground state properties; calculation and simulation; regulatory system;

\begin{abstract}
In radiation damage research, copper, nickel, and their alloys are widely used model systems for face-centered-cubic (FCC) metals. The ground-states properties of the ordered and disordered alloys are studied by using the molecular dynamics (MD) and first-principles calculations. For copper-nickel alloys, the equilibrium properties have been predicted by large-scale atomic/molecular massively parallel simulator (LAMMPS), Abinit and WIEN2k, thermodynamics is calculated by LAMMPS and Abinit. In order to investigate the disordered alloys, special quasirandom structures (SQS) and fractional function are adopted in LAMMPS, and their capabilities are demonstrated to predict the properties of disordered alloys. For both the ordered and disordered alloys, the lattice constants in agreement with the Vegard's law are predicted and the bulk moduli present the deviations with respect to the experimental values; with the increasing weight concentration of nickel, the equilibrium volumes reduce and the bulk moduli increase. The calculated cohesive energy of copper and nickel are consistent with the experimental values; the cohesive energies of ordered alloys are predicted. The energies of formation of disordered alloys are always lower than those of ordered alloys.
\end{abstract}

\section{Introduction}

In the past decades, researchers have paid huge efforts to study the structure and theoretical models of the random (disordered) alloys (e.g. SQS), because one would like to develop a complete or substitutional structure and theoretical model for the disordered alloy. However, owing to uncertainty about atomic occupations in the crystal lattice, which is difficult to determine the positions of $\mathrm{A}$ and $\mathrm{B}$ atoms in a disordered alloy $A_{1-x} B_{x}$, many problems are still existed in it, for instance, lowering the total energy of the disordered alloy in the calculation ${ }^{[1]}$, only dealing with certain specific components of the disordered alloy ${ }^{[2]}$, etc.

\section{Theory and methodology}

\subsection{Disordered and ordered structures}

We employ the alloy theoretic automated toolkit (ATAT) code ${ }^{[3]}$ to investigate all the possible configurations and generate the FCC-based four-, eight-, and 16-atom SQSs with the composition $\mathrm{x}$ $=0.25,0.5$ and 0.75 for the disordered alloys. In the MD simulations, large-scale atomic/molecular massively parallel simulator ${ }^{[4]}$ is employed. In order to get the disordered (random) structures in LAMMPS, we set the atom type for a fraction of the selected atoms. As the calculated result is statistically close, the error control of the atom number is 1 or so under identical random number. In the case of the ordered structure, we mainly consider that three ordered alloys are $\mathrm{Cu}_{3} \mathrm{Ni}\left(\mathrm{L}_{2}\right)$, $\mathrm{CuNi}\left(\mathrm{L}_{0}\right)$, and $\mathrm{CuNi}_{3}\left(\mathrm{~L}_{2}\right)$, respectively, and two elements being $\mathrm{Cu}$ and $\mathrm{Ni}(\mathrm{A} 1)$. 


\subsection{MD and first-principles calculations}

In the present work of MD simulations, $\mathrm{Cu}-\mathrm{Ni}$ alloy potential is generated by the EAM database tool (Written by Xiaowang Zhou) based on the copper and nickel parameters ${ }^{[5]}$. Our first-principles calculations employ the Abinit code ${ }^{[6]}$ with ion-electron interaction described by the projector augmented-paw (PAW) method ${ }^{[7]}$. For fitting the MD calculated cohesive energy versus lattice parameter to bulk modulus, second-order polynomial fit by the program gnuplot or the software Origin is used. According to the definition of bulk modulus $\mathrm{B}_{0}, B_{0}=-\frac{d P}{d V / V}$. where $\mathrm{P}$ and $\mathrm{V}$ are pressure and volume of the unit cell, respectively. For the cubic unit cell, $P=-\frac{d w}{d V}=-\frac{N}{3 a^{2}} \frac{d E}{d a}$, where $\mathrm{N}$ and $a$ are atom number and lattice constant of the unit cell, respectively. Thus, bulk modulus is $B_{0}=\left.\frac{N}{9 a_{0}} \frac{d^{2} E}{d a^{2}}\right|_{a_{0}}$, where $a_{0}$ is equilibrium lattice constant. In order to fit the firstprinciples calculated energy-vs-volume data to Murnaghan equation of state (EOS), we use the program ev.x (Contributions by Eyvaz Isaev) in the Quantum ESPRESSO ${ }^{[8]}$. The equilibrium properties calculated by the above EOS include the equilibrium volume $V_{0}$, bulk modulus $B_{0}$, and its pressure derivative $B_{0}^{\prime}$. In the paper, we present the fitting results of lattice constants and bulk modulus of $\mathrm{Cu}$ and $\mathrm{Ni}$ in Table 1 with available experimental data.

\section{Results and discussions}

From Table 1, the lattice constants and bulk moduli fitted by polynomial and EOS for the FCC $\mathrm{Cu}$ and FCC Ni in comparison with experimental values ${ }^{[9-11]}$ are summarized. It is apparent that the GGA (rather than LDA) describes the FCC Cu and FCC Ni very well. In first-principles calculation, lattice constants and bulk moduli calculated by the GGA potential are in better agreement with experimental values. Whereas in the MD calculation, lattice constants are in good agreement with experimental values, but its bulk moduli are not in good agreement with the corresponding experimental values. Hence, the GGA potential is used in first-principles calculation.

Table 1. Lattice constants of FCC copper and FCC nickel calculated by molecular dynamics (MD) and first-principles, and then bulk moduli of FCC copper and FCC nickel fitted by polynomial and

EOS, respectively, where the percent error between the calculated values and the experimental values are shown in the parentheses

\begin{tabular}{cccccc}
\hline \hline & Metal & MD & GGA & LDA & $\begin{array}{c}\text { Experimental } \\
\text { value }\end{array}$ \\
\hline Lattice constant & $\mathrm{Cu}$ & 3.615 & 3.651 & 3.535 & $3.615^{\mathrm{a}}$ \\
(Angstrom) and & $(\mathrm{FCC})$ & $(0)$ & $(1 \%)$ & $(-2.2 \%)$ & \\
error & $\mathrm{Ni}$ & 3.520 & 3.529 & 3.429 & $3.520^{\mathrm{b}}$ \\
& $(\mathrm{FCC})$ & $(0)$ & $(0.26 \%)$ & $(-2.6 \%)$ & \\
Bulk modulus & $\mathrm{Cu}$ & 129.8 & 130.7 & 178.6 & $137.0^{\mathrm{a}}$ \\
$(\mathrm{FCC})$ & $(-5.3 \%)$ & $(-4.6 \%)$ & $(30.4 \%)$ & \\
& $\mathrm{Ni}$ & 218.6 & 187.8 & 242.1 & $186.0^{\mathrm{b}}$ \\
\hline \hline
\end{tabular}

a Reference 9.

b References 10 and 11.

\subsection{Equilibrium properties}

The equilibrium lattice constants of copper and nickel by LAMMPS calculation in Fig. 1 are in line with the experimental values ${ }^{[12]}$. According to the Vegard's law, the lattice constants of the ideal mixing alloys can be written as $a(x)=(1-x) \cdot a_{C u}+x \cdot a_{N i}$. By calculations, we find that our calculated lattice constants of the ordered alloys (see Fig. 1) agree well with the Vegard's law. But the calculated bulk modulus of copper and nickel are quite different from the experimental values 
(see Table 2).

The comparison of the cohesive energy of the pure metals with that of the alloys is needed in any prediction about the phase diagram of binary alloys. In MD calculation, the definition of the cohesive energy is a minimum of the crystal lattice energy. We calculate the cohesive energy of copper and nickel, whose corresponding values are $-3.54 \mathrm{eV}$ per atom and $-4.45 \mathrm{eV}$ per atom as presented in Fig. 1, while the cohesive energy of copper is $-3.50 \mathrm{eV}$ per atom in the experiment.

Vacancy formation energy is defined by the following expression. $E_{v}=E_{\text {relax }}-(N-1) E_{c o h}$, where $E_{v}, E_{\text {relax }}$, and $E_{\text {coh }}$ express the vacancy formation energy, the crystal energy including $\mathrm{N}-1$ atoms after relaxation, and the cohesive energy, respectively; $\mathrm{N}$ is atom numbers of the unit cell in $\mathrm{MD}$ calculation. Our calculated vacancy formation energies of copper and nickel are $1.28 \mathrm{eV}$ and $1.63 \mathrm{eV}$, which are consistent with the corresponding calculated values ${ }^{[13]}$ and experimental values $^{[14,15]}$.

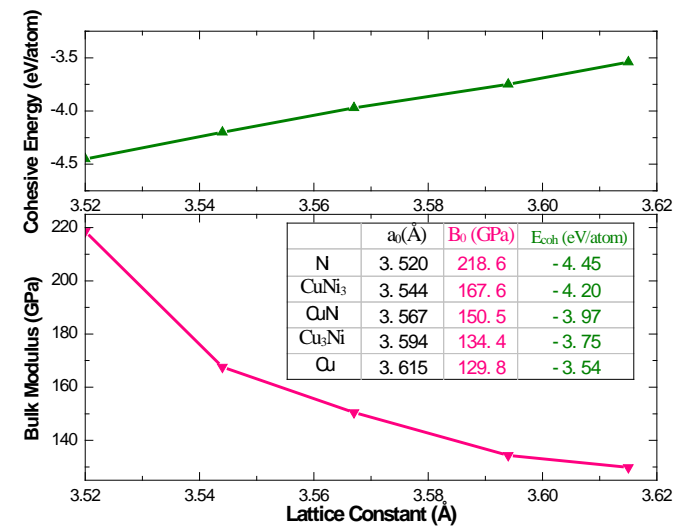

Fig. 1. (Color online) The equilibrium lattice constant, the bulk modulus and the cohesive energy of copper, nickel and their ordered alloys by MD calculation and second-order polynomial fitting

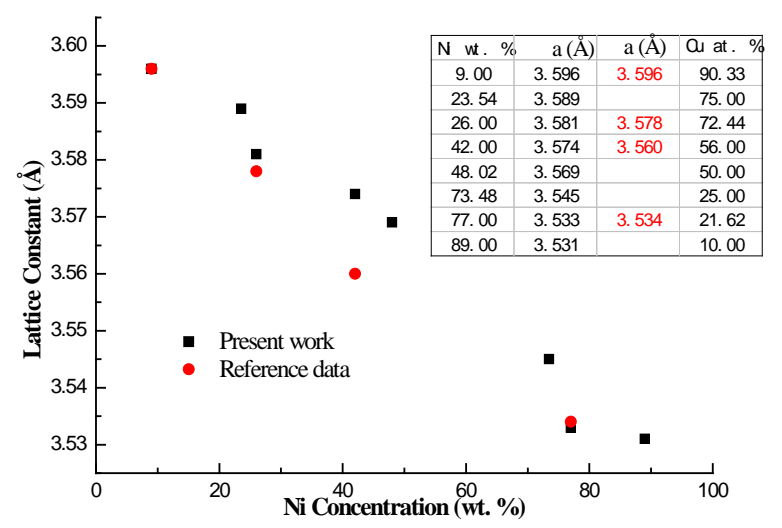

Fig. 2. (Color online) The lattice constant of different weight concentration calculated by LAMMPS. Red data from reference ${ }^{[16]}$

We calculate the lattice constants of the random alloys of copper and nickel by LAMMPS in Fig. 2. The lattice constant of the random alloys presents a downward trend with increasing nickel concentration. And compared with the experimental values $\left.{ }^{[16}\right]$ of the same nickel weight concentration, our calculated lattice constants of $9 \%, 26 \%$, and $77 \%$ agree well, while the lattice constants of $42 \%$ nickel weight concentration has a small deviation. From Fig. 2, we can know that the lattice constants of the weight concentration of the random copper and nickel alloys are in good agreement with those of the corresponding weight or atom concentration of the ordered copper and nickel alloys (see Fig. 1). This can probably get an explanation from unlimited miscibility and relatively close lattice constants of copper and nickel.

In Abinit calculation, the equilibrium volumes reduce with the increasing weight concentration of nickel whether its structure is the ordered or not, and inversely the bulk moduli increase with the increasing weight concentration of nickel as showed in Table 2, i.e. the larger the volume, the 
smaller the bulk modulus. However, WIEN2k calculations under nonmagnetic states present the same trends as Abinit's in terms of the equilibrium volumes and bulk moduli as showed in Table 3. At the same time the equilibrium volumes calculated by Abinit are larger than by WIEN2k, whereas bulk moduli by WIEN2k are larger than by Abinit. The total energies calculated by WIEN2k higher than by Abinit are the best explanation. This is due to the fact that WIEN2k uses all-electron potential considering the contribution of core electrons to the total energy whereas pseudopotential's is not considered. However, the pressure derivatives do not present the same properties as the equilibrium volumes and bulk moduli. In WIEN2k calculation under nonmagnetic states, the equilibrium volume and pressure derivative of CuNi alloy ( $\left.\mathrm{L} 1_{0}\right)$ are the smallest (see Table 3) compared with those of the other structures.

Table 2. Together with the first-principles properties using the Abinit code at the ground state fitted by the Murnaghan EOS, including the equilibrium volume $\mathrm{V}_{0}\left(\AA^{3}\right.$ per atom), bulk modulus $\mathrm{B}_{0}$

$(\mathrm{GPa})$, its pressure derivative $B_{0}^{\prime}$, and the equilibrium energy $\mathrm{E}_{0}(\mathrm{eV})$

\begin{tabular}{ccccccc}
\hline \hline Entry & Name & Formula & $\mathrm{V}_{0}$ & $\mathrm{~B}_{0}$ & $B_{0}^{\prime}$ & $\mathrm{E}_{0}$ \\
\hline 1 & $\mathrm{~A} 1$ & $\mathrm{Cu}$ & 12.18 & 130.7 & 5.07 & -1395.52 \\
2 & $\mathrm{~A} 1$ & $\mathrm{Ni}$ & 10.99 & 187.8 & 4.19 & -3508.03 \\
3 & $\mathrm{~L} 1_{2}$ & $\mathrm{Cu}_{3} \mathrm{Ni}$ & 11.85 & 142.5 & 5.18 & -7694.50 \\
4 & $\mathrm{~L} 1_{0}$ & $\mathrm{CuNi}$ & 11.48 & 159.5 & 4.55 & -4903.47 \\
5 & $\mathrm{~L} 1{ }_{2}$ & $\mathrm{CuNi}_{3}$ & 11.27 & 170.9 & 4.92 & -11919.48 \\
6 & $\mathrm{SQS}-4$ & $\mathrm{Cu}_{3} \mathrm{Ni}$ & 11.84 & 143.4 & 4.78 & -7694.62 \\
7 & $\mathrm{SQS}-4$ & $\mathrm{Cu}_{2} \mathrm{Ni}_{2}$ & 11.54 & 157.8 & 4.66 & -9807.21 \\
8 & $\mathrm{SQS}-4$ & $\mathrm{CuNi}_{3}$ & 11.25 & 172.7 & 4.65 & -11919.84 \\
9 & $\mathrm{SQS}-8$ & $\mathrm{Cu}_{6} \mathrm{Ni}_{2}$ & 11.84 & 143.7 & 4.94 & -15389.25 \\
10 & $\mathrm{SQS}-8$ & $\mathrm{Cu}_{4} \mathrm{Ni}_{4}$ & 11.54 & 159.2 & 3.37 & -19614.41 \\
11 & $\mathrm{SQS}-8$ & $\mathrm{Cu}_{2} \mathrm{Ni}_{6}$ & 11.25 & 173.2 & 5.11 & -23839.67 \\
12 & $\mathrm{SQS}-16$ & $\mathrm{Cu}_{12} \mathrm{Ni}_{4}$ & 11.84 & 144.7 & 5.49 & -30778.51 \\
13 & $\mathrm{SQS}-16$ & $\mathrm{Cu}_{8} \mathrm{Ni}_{8}$ & 11.53 & 160.4 & 3.71 & -39228.83 \\
14 & $\mathrm{SQS}-16$ & $\mathrm{Cu}_{4} \mathrm{Ni}_{12}$ & 11.25 & 173.2 & 5.23 & -47679.35 \\
\hline \hline
\end{tabular}

Table 3. Together with the first-principles properties using the WIEN2k code at the ground state fitted by the Murnaghan EOS, including the equilibrium volume $\mathrm{V}_{0}\left(\AA^{3}\right.$ per atom), bulk modulus $\mathrm{B}_{0}$ (GPa) and its pressure derivative $B_{0}^{\prime}$

\begin{tabular}{|c|c|c|c|c|c|c|}
\hline Entry & Name & Formula & State & $\mathrm{V}_{0}$ & $\mathrm{~B}_{0}$ & $\overline{B_{0}^{\prime}}$ \\
\hline 1 & A1 & $\mathrm{Cu}$ & NM & 11.77 & 151.7 & 4.34 \\
\hline \multirow[t]{2}{*}{2} & A1 & $\mathrm{Ni}$ & NM & 10.70 & 219.6 & 6.85 \\
\hline & & & FM & 10.78 & 214.9 & 3.96 \\
\hline \multirow[t]{2}{*}{3} & $\mathrm{~L} 1_{2}$ & $\mathrm{Cu}_{3} \mathrm{Ni}$ & NM & 11.48 & 165.6 & 3.08 \\
\hline & & & FM & 11.46 & 171.6 & 4.20 \\
\hline \multirow[t]{2}{*}{4} & $\mathrm{~L} 1_{0}$ & $\mathrm{CuNi}$ & NM & 11.30 & 194.3 & 1.31 \\
\hline & & & FM & 11.26 & 176.7 & 5.27 \\
\hline \multirow[t]{2}{*}{5} & $\mathrm{~L} 1_{2}$ & $\mathrm{CuNi}_{3}$ & NM & 10.92 & 202.3 & 6.08 \\
\hline & & & FM & 11.00 & 175.6 & 6.10 \\
\hline \multirow[t]{2}{*}{6} & SQS-4 & $\mathrm{Cu}_{3} \mathrm{Ni}$ & NM & 11.43 & 165.2 & 5.46 \\
\hline & & & FM & 11.45 & 164.3 & 5.10 \\
\hline \multirow[t]{2}{*}{7} & SQS-4 & $\mathrm{Cu}_{2} \mathrm{Ni}_{2}$ & NM & 11.17 & 172.8 & 7.15 \\
\hline & & & FM & 11.19 & 182.1 & 4.60 \\
\hline \multirow[t]{2}{*}{8} & SQS-4 & $\mathrm{CuNi}_{3}$ & NM & 10.93 & 184.7 & 6.95 \\
\hline & & & FM & 11.04 & 191.2 & 3.01 \\
\hline
\end{tabular}

\subsection{Thermodynamics}

Energy of formation is one of important basis that determine the stability of crystal structure. For ordered alloys, the energy of formation is given by $E_{f}^{n}=E\left(C u_{1-x} N i_{x}\right)-(1-x) E(C u)-x E(N i)$, where $E_{f}^{n}$ corresponds to the energy of formation of each of the above five ordered structures, in units of eV/atom. For disordered alloys, their energy of formation can be obtained as the following expression ${ }^{[17]}$ by using a random distribution for statistical averaging. 
$E_{f}(x)=\sum_{n=0}^{4} P_{n}(x) E_{f}^{n}$, where $E_{f}(x)$ is the energy of formation of disordered alloys and $P_{n}(x)$ is the statistical weight. The weight expresses the probability that the $n$th short-range ordered structure appears in the alloy and can be represented as $P_{n}(x)=\left(\begin{array}{l}4 \\ n\end{array}\right) x^{n}(1-x)^{4-n}$.

Therefore, for LAMMPS calculation, the energy of formation of the disordered alloys by above the method can be expanded as the following expression: $E_{f}(x)=0.13 x^{4}-0.42 x^{3}+0.36 x^{2}+0.07 x$. According to the expressions 7 and 10, we calculate the energy of formation by LAMMPS and Abinit for disordered alloys as shown in Figs. 3 and 4. The energies of formation of disordered $\mathrm{Cu}_{1-x} \mathrm{Ni}_{x}$ alloys are always lower than those of ordered $\mathrm{Cu}_{1-x} \mathrm{Ni}_{x}$ alloys (see Figs. 3 and 4). This trend indicates that the similar results are obtained by MD and first-principles calculations but the values calculated by Abinit are greater than by LAMMPS.

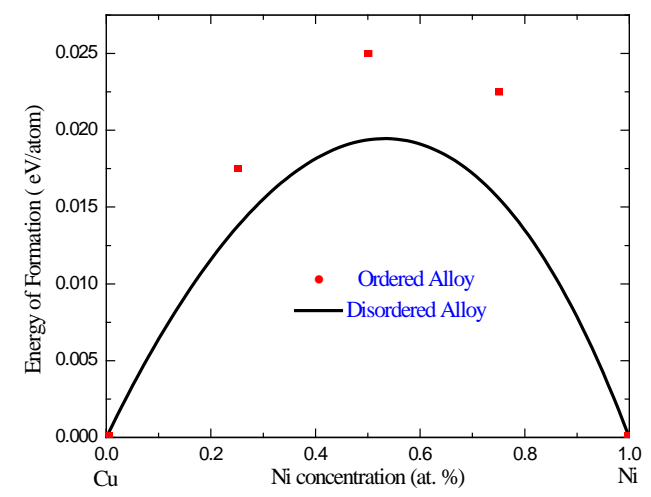

Fig. 3. (Color online) The energy of formation of the disordered alloys as a function of nickel concentration (by LAMMPS)

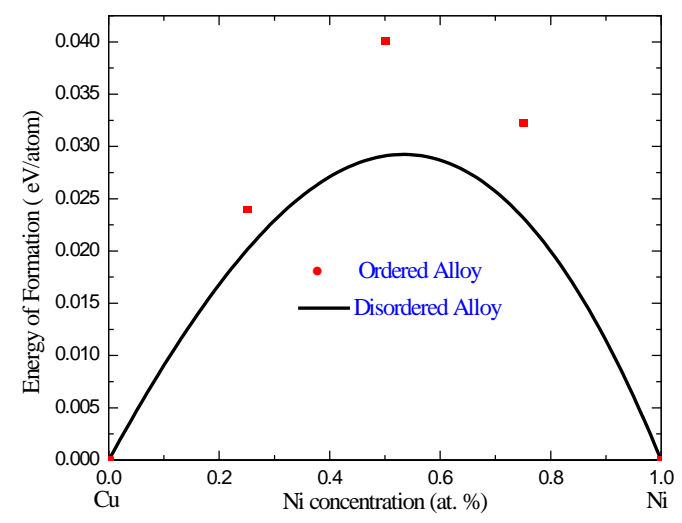

Fig. 4. (Color online) The energy of formation of the disordered alloys as a function of nickel concentration (by Abinit)

\section{Conclusion}

In order to investigate the disordered alloys, we demonstrate their capabilities to predict the properties of disordered alloys including equilibrium properties, and thermodynamics. For both the ordered and disordered $\mathrm{Cu}_{1-x} N i_{x}$ alloys, it is found in the present work that (i) the lattice constants in agreement with the Vegard's law are predicted and the bulk moduli present the deviations with respect to the experimental values; (ii) with the increasing weight concentration of nickel, the equilibrium volumes reduce and the bulk moduli increase, i.e. the larger the volume, the smaller the bulk modulus; and (iii) the calculated cohesive energy of copper and nickel are consistent with the experiment values; the cohesive energies of ordered $\mathrm{Cu}_{1-x} \mathrm{Ni}_{x}$ alloys are predicted. By calculating the 
energies of formation of ordered $\mathrm{Cu}_{1-x} \mathrm{Ni}_{x}$ alloys, the gotten energies of formation of disordered alloys are always lower than those of ordered alloys.

\section{Acknowledgement}

Fujian province middle and young teacher education research project (No. JAT160303).

\section{References}

[1] WEI S H, FERREIRA L G, BERNARD J E,et al. Electronic properties of random alloys: Special quasirandom structures [J]. Physical Review B Condensed Matter, 1990, 42 (15):9622.

[2] CONNOLLY J W D, WILLIAMS A R. Density-functional theory applied to phase transformations in transition-metal alloys [J]. Physical Review B Condensed Matter, 1983, 27 (8):5169-5172.

[3] WALLEVAN de A. Multicomponent multisublattice alloys, nonconfigurational entropy and other additions to the Alloy Theoretic Automated Toolkit [J]. Calphad-computer Coupling of Phase Diagrams\&Thermochemistry, 2009, 33 (2):266-278.

[4] PLIMPTON S. Fast parallel algorithms for short-range molecular dynamics [J].Journal of Computational Physics, 1995, 117:1-19.

[5] ZHOUX W, JOHNSON R A, WADLEY H N G. Misfit-energy-increasing dislocations in vapor-deposited CoFe/NiFe multilayers [J]. Physical Review B, 2004, 69 (14):1124-1133.

[6] TORRENt M, JOLLET F, BOTTIN F, et al. Implementation of the projector augmented-wave method in the ABINIT code: Application to the study of iron under pressure [J]. Computational Materials Science, 2008, 42 (2):337-351.

[7] KRESSE G, JOUBERT D. From ultrasoft pseudopotentials to the projector augmented-wave method [J]. Physical Review B, 1999, 59 (3):1758-1775.

[8] GIANNOZZI P, BARONI S, BONINI N, et al. QUANTUM ESPRESSO: a modular and opensource software project for quantum simulations of materials. [J]. Journal of Physics: Condensed Matter, 2009, 21 (39):395502.

[9] BALLUFFI R W. Vacancy defect mobilities and binding energies obtained from annealing studies [J]. Journal of Nuclear Materials, 1978, 69-70 (69):240-263.

[10] WYCISK W, KNIEPMEIER M. Quenching experiments in high purity Ni [J]. Journal of Nuclear Materials, 1978, 69-70 (69):616-619.

[11] STRAUMAL B B, PROTASOVA S G, MAZILKIN A A, et al. Deformation-driven formation of equilibrium phases in the $\mathrm{Cu}-\mathrm{Ni}$ alloys [J]. Journal of Materials Science, 2012, 47 (1):360-367.

[12] LAMBRECHT W R, SEGALL B. Anomalous band-gap behavior and phase stability of c-BNdiamond alloys [J].Physical Review B, 1993, 47 (47):9289-9296.

[13] KikuchiR.A theory of cooperative phenomena [J]. Physical Review, 1951, 81 (81):988.

[14] KIKUCHI R. Superposition approximation and natural iteration calculation in cluster variation method [J]. Journal of Chemical Physics, 2003, 60 (3):1071-1080.

[15] SANCHEZ J M, FONTAINE de D. The fee Ising model in the cluster variation approximation [J].Physical Review B, 1978, 17 (17):2926-2936.

[16] ZHENG J C, HUAN C H A, WEE A T S, et al. Ground-state properties of cubic C-BN solid solutions [J]. Journal of Physics Condensed Matter, 1999, 11 (3):927.

[17] DONOHUE J. The structures of the elements [M]. New York: Wiley, 1974: 191-199. 